\title{
Hyperbolic Transformation and Average Elasticity in the Framework of the Fixed Effects Logit Model
}

\author{
Yoshitsugu Kitazawa \\ Faculty of Economics, Kyushu Sangyo University, Fukuoka, Japan \\ Email: kitazawa@ip.kyusan-u.ac.jp
}

Received January 13, 2012; revised February 11, 2012; accepted February 20, 2012

\begin{abstract}
In this paper, a simple transformation is proposed for the fixed effects logit model, which constructs some valid moment conditions including the first-order condition for one of the conditional MLE proposed by Chamberlain (1980) [1]. Some Monte Carlo experiments are carried out for the GMM estimator based on the transformation. In addition, the average elasticity of the logit probability with respect to the exponential function of explanatory variable is proposed in the framework of the fixed effects logit model, which is computable without the fixed effects.
\end{abstract}

Keywords: Fixed Effects Logit; Conditional Logit Estimator; Hyperbolic Transformation; Moment Conditions; GMM; Monte Carlo Experiments; Average Elasticity

\section{Introduction}

Chamberlain (1980) [1] proposes the useful and established estimator for the fixed effects logit model in panel data. ${ }^{1}$ This estimator is referred to as the conditional logit estimator, which maximizes the likelihood function composed of the probabilities of the (binary) dependent variables conditional on the fixed effects, the (real-valued) explanatory variables, and the intertemporal sums of the dependent variables. The conditional logit estimator is consistent for the situation of small number of time periods and large cross-sectional size, since its conditional likelihood function rules out the fixed effects and accordingly circumvents the incidental parameters problems pointed out by Neyman and Scott (1948) [2]. ${ }^{2}$

This paper advocates another method of consistently estimating the fixed effects logit model for the situation of small number of time periods and large cross-sectional size. ${ }^{3}$ The procedure of the method is as follows: First, a hyperbolic transformation is applied to the fixed effects logit model with the aim of eliminating the fixed effects. Next, the GMM (generalized method of moments) estimator proposed by Hansen (1982) [20] is constructed by using the moment conditions based on the hyperbolic transformation. It will be seen that these moment conditions include one type of the first-order conditions of the

\footnotetext{
${ }^{1}$ The rootstock of this estimator is Rasch (1960) [3], (1961) [4].

${ }^{2}$ Additionally, Honoré and Kyriazidou (2000) [5] propose an estimator for the fixed effects logit model with the lagged dependent variable (as for details, see also pp. 211-216 in Hsiao, 2003 [6]). Further, Thomas (2006) [7] proposes two estimators for the fixed effects logit model with heterogeneous linear trends.
}

likelihood for the conditional logit estimator. Then, the preferable small sample property of the GMM estimator using the moment conditions based on the hyperbolic transformation is shown by some Monte Carlo experiments.

In addition, this paper presents the calculation formula of the average elasticity of the logit probability with respect to the exponential function of explanatory variable for the fixed effects logit model. The average marginal effect is not obtained due to the incidental parameters problems for the case of the fixed effects logit model with time dimension being strictly fixed, while it seems that no appropriate index measuring the effect of the change of explanatory variable is developed, in author's best knowledge. Since the average elasticity is able to be calculated using the consistent estimator of the parameter of interest and the average of binary dependent variables without relation to the fixed effects, it can be said that it is a revolutionary index for the fixed effects logit model.

\footnotetext{
${ }^{3}$ It seems that the mainstream of late is the development of the biasadjusted estimators, which is available in nonlinear panel data models and aims at the reduction of time-series finite sample bias (i.e. the approximately unbiased estimation of the incidental parameters as well as the parameters of interest, leading to obtaining the approximate marginal effects). Various approaches are proposed in line with the bias-adjustment: Hahn and Newey (2004) [8], Cox and Reid (1987) [9], Lancaster (2002) [10], Arellano (2003) [11], Arellano and Bonhomme (2009) [12], Carro (2007) [13], Fernández-Val (2009) [14], Severini (1998) [15], Pace and Salvan (2006) [16], Bester and Hansen (2009) [17], etc. Some of the approaches are reviewed in Arellano and Hahn (2007) [18] and Hsiao (2010) [19]. However, author's policy is to conduct the consistent estimation for the case of small time dimension and therefore this paper is not bent upon the bias-adjusted estimators.
} 
The rest of the paper is as follows: Section 2 presents the implicit form of the fixed effects logit model, the moment conditions based on the hyperbolic transformation, and the GMM estimator. Section 3 illustrates the link between the conditional maximum likelihood estimator (CMLE) mentioned in the first paragraph in this section and the GMM estimator for the case of two periods. Section 4 reports some Monte Carlo results for the GMM estimator. Section 5 presents the average elasticity in the framework of the fixed effects logit model. Section 6 concludes.

\section{Fixed Effects Logit Model, Hyperbolic Transformation and GMM Estimator}

In this section, the (static) fixed effects logit model is implicitly defined where the error term is of additive form. ${ }^{4}$ The hyperbolic transformation, which eliminates the fixed effects and then based on which the moment conditions is constructed for estimating the model consistently, is the fruits of the model defined implicitly. The GMM estimator is defined by using the moment conditions constructed. Throughout this paper, the subscripts $i$ and $t$ denote the individual and time period respectively, while $N$ and $T$ are number of individuals and number of time periods respectively. Since the short panel is supposed, it is assumed that $N \rightarrow \infty$ and $T$ is fixed. In addition, it is assumed that the variables in the model are independent among individuals.

The fixed effects logit model is able to be written in the implicit form as follows:

$$
\begin{aligned}
& y_{i t}=p_{i t}+v_{i t}, \text { for } t=1, \cdots, T, \\
& p_{i t}=\exp \left(\psi_{i}+\delta w_{i t}\right) /\left(1+\exp \left(\psi_{i}+\delta w_{i t}\right)\right), \\
& \text { for } t=1, \cdots, T
\end{aligned}
$$

where the observable variables $y_{i t}$ and $w_{i t}$ are the binary dependent variable and the real-valued explanatory variable respectively, while the unobservable variables $\psi_{i}$ and $v_{i t}$ are the individual fixed effect and the disturbance respectively. ${ }^{5}$ Equations (2.1) say that $y_{i t}$ take one with probability $p_{i t}$, while it is seen from Equations (2.2) that the probability is the logistic cumulative distribution function of $\psi_{i}+\delta w_{i t}$. Allowing for the serially uncorrelated disturbances, the uncorrelatedness between the disturbances and the fixed effect and the strictly exogenous explanatory variables, the assumptions on the disturbances are specified as

$$
\mathrm{E}\left[v_{i t} \mid v_{i}^{t-1}, \psi_{i}, w_{i}^{T}\right]=0, \text { for } t=1, \cdots, T,
$$

where $v_{i}^{t-1}=\left(v_{i 1}, \cdots, v_{i, t-1}\right)$ for $t=2, \cdots, T, \quad v_{i}^{0}$ is de-

${ }^{4}$ The regression form defined implicitly is also used by Blundell et al. (2002) [21] for count panel data.

${ }^{5}$ It is generally assumed that the individual effect $\psi_{i}$ is correlated with the explanatory variables $w_{i t}$ for each $i$. fined as the empty set for convenience and $w_{i}^{T}=\left(w_{i 1}, \cdots, w_{i T}\right)$. The assumptions $(2.3)$ can be derived from the assumption underlying the fixed effects logit model, which is that $y_{i t}$ for $t=1, \cdots, T$ are mutually independent conditional on $\psi_{i}$ and $w_{i}^{T}{ }^{6}$

From now on, based on the fixed effects logit model composed of (2.1) and (2.2) with (2.3), the moment conditions for estimating the parameter of interest $\delta$ consistently are constructed by using a hyperbolic transformation, as stated below. Taking notice of the fact that

$$
\tanh \left(\left(\psi_{i}+\delta w_{i t}\right) / 2\right)=2 p_{i t}-1
$$

and using the formula that

$$
\begin{aligned}
& \tanh (a-b) \\
& =(\tanh (a)-\tanh (b)) /(1-\tanh (a) \tanh (b))
\end{aligned}
$$

with $a$ and $b$ being any real numbers, it follows that

$$
\begin{aligned}
& \tanh \left(\delta \Delta w_{i t} / 2\right) \\
& =\left(p_{i t}-p_{i, t-1}\right) /\left(p_{i t}+p_{i, t-1}-2 p_{i t} p_{i, t-1}\right),
\end{aligned}
$$

where $\Delta$ is the first differencing operator, such as $\Delta w_{i t}=w_{i t}-w_{i, t-1}$. Since $p_{i t}$ and $p_{i t} p_{i, t-1}$ are written as

$$
p_{i t}=\mathrm{E}\left[y_{i t} \mid v_{i}^{t-1}, \psi_{i}, w_{i}^{T}\right]
$$

and

$$
p_{i t} p_{i, t-1}=\mathrm{E}\left[y_{i t} y_{i, t-1} \mid v_{i}^{t-1}, \psi_{i}, w_{i}^{T}\right]-p_{i t} v_{i, t-1}
$$

respectively by using (2.1) and (2.3), plugging (2.7) and (2.8) into (2.6) gives

$$
\begin{aligned}
& \left(\mathrm{E}\left[y_{i t} \mid v_{i}^{t-1}, \psi_{i}, w_{i}^{T}\right]+\mathrm{E}\left[y_{i, t-1} \mid v_{i}^{t-2}, \psi_{i}, w_{i}^{T}\right]\right. \\
& \left.-2\left(\mathrm{E}\left[y_{i t} y_{i, t-1} \mid v_{i}^{t-1}, \psi_{i}, w_{i}^{T}\right]-p_{i t} v_{i, t-1}\right)\right) \tanh \left(\delta \Delta w_{i t} / 2\right) \\
& =\mathrm{E}\left[y_{i t} \mid v_{i}^{t-1}, \psi_{i}, w_{i}^{T}\right]-\mathrm{E}\left[y_{i, t-1} \mid v_{i}^{t-2}, \psi_{i}, w_{i}^{T}\right] .
\end{aligned}
$$

Equations (2.7) and (2.8) are obtained by plugging (2.1) into $\mathrm{E}\left[y_{i t} \mid v_{i}^{t-1}, \psi_{i}, w_{i}^{T}\right]$ and $\mathrm{E}\left[y_{i t} y_{i, t-1} \mid v_{i}^{t-1}, \psi_{i}, w_{i}^{T}\right]$ and then applying (2.3) to them. Taking the expectation conditional on $\left(v_{i}^{t-2}, \psi_{i}, w_{i}^{T}\right)$ for both sides of (2.9) and then applying law of iterated expectation and (2.3) dated $t-1$, it follows that

\footnotetext{
${ }^{6}$ If the underlying assumption holds, the following relationship is obtained: $f\left(y_{i t} \mid y_{i}^{t-1}, \psi_{i}, w_{i}^{T}\right)=f\left(y_{i t} \mid \psi_{i}, w_{i}^{T}\right)=p_{i t}$, where $f(\bullet \bullet)$ is the conditional probability density function and $y_{i}^{t-1}=\left(y_{i 1}, \cdots, y_{i, t-1}\right)$. Accordingly, it follows that $\mathrm{E}\left[y_{i t} \mid y_{i}^{t-1}, \psi_{i}, w_{i}^{T}\right]=\mathrm{E}\left[y_{i t} \mid \psi_{i}, w_{i}^{T}\right]=p_{i t}$. As for details, see p. 23 in Cameron and Trivedi (2005) [22]. Taking notice of (2.1) and the fact that $v_{i t}=y_{i t}-p_{i t}$, the assumptions (2.3) are obtained.
} 


$$
\mathrm{E}\left[\left(\left(y_{i t}-y_{i, t-1}\right)-\tanh \left(\delta \Delta w_{i t} / 2\right)\left(y_{i t}+y_{i, t-1}-2 y_{i t} y_{i, t-1}\right)\right) \mid v_{i}^{t-2}, \psi_{i}, w_{i}^{T}\right]=0 .
$$

Since $\left(y_{i t}\right)^{n}=y_{i t}$ for any positive integer value $n$ due to the property of binary variable (and accordingly

$$
\mathrm{E}\left[h_{i t}(\delta) \mid v_{i}^{t-2}, \psi_{i}, w_{i}^{T}\right]=0, \text { for } t=2, \cdots, T,
$$

where

$$
h_{i t}(\delta)=\Delta y_{i t}-\tanh \left(\delta \Delta w_{i t} / 2\right)\left(\Delta y_{i t}\right)^{2} .
$$

The transformation (2.12) is referred to as "the hyperbolic tangent differencing transformation" for the fixed effects logit model in this paper and hereafter abbreviated to "the HTD transformation". It should be noted that as seen from (2.11) and (2.12), observations for which $y_{i t}=y_{i, t-1}=0$ and $y_{i t}=y_{i, t-1}=1$ make no direct contribution to obtaining the estimates of $\delta$ based on the moment conditions (2.11), since $h_{i t}(\delta)$ is invariably zero for these observations.

The conditional moment conditions (2.11) give the following $m \times 1$ vector of unconditional moment conditions:

$$
\mathrm{E}\left[\left(z_{i}\right)^{\prime} h_{i}(\delta)\right]=0
$$

where $h_{i}(\delta)=\left[h_{i 2}(\delta) \cdots h_{i T}(\delta)\right]^{\prime}$ is the $(T-1) \times 1$ vector and $z_{i}=\operatorname{diag}\left[\left(z_{i 2}\right)^{\prime} \cdots\left(z_{i T}\right)^{\prime}\right]$ is the $(T-1) \times m$

$$
N^{1 / 2}\left(\hat{\delta}_{G M M}-\delta_{0}\right) \stackrel{d}{\rightarrow} \mathrm{N}\left(0,\left(\left(D\left(\delta_{0}\right)\right)^{\prime}\left(\Omega\left(\delta_{0}\right)\right)^{-1} D\left(\delta_{0}\right)\right)^{-1}\right)
$$

with $\delta_{0}$ being the true value of $\delta$. Taking notice of the assumption that the variables are independent among individuals and adding the assumption that the variables are identically distributed among individuals, $\Omega\left(\delta_{0}\right)$, which is the (asymptotic) variance-covariance matrix of

${ }^{7}$ If the much weaker assumptions $\mathrm{E}\left[v_{i t} \mid v_{i}^{t-1}, \psi_{i}, w_{i}^{t+1}\right]=0$ for $t=1, \cdots, T$ are used instead of (2.3), the moment conditions $\mathrm{E}\left[h_{i t}(\delta) \mid v_{i}^{t-2}, \psi_{i}, w_{i}^{t}\right]=0$ for $t=2, \cdots, T$ can be obtained instead of (2.11), where $w_{i}^{t}=\left(w_{i 1}, \cdots, w_{i t}\right)$ and $w_{i, T+1}$ is defined as the empty set for convenience. It should be noted that under the assumptions $\mathrm{E}\left[v_{i t} \mid v_{i}^{t-1}, \psi_{i}, w_{i}^{t+1}\right]=0$ for $t=1, \cdots, T$, the (consistent) CMLE proposed by Chamberlain (1980) [1] is no longer obtained for $T \geq 3$. The implication of $\mathrm{E}\left[v_{i t} \mid v_{i}^{t-1}, \psi_{i}, w_{i}^{t+1}\right]=0$ is that although the decision $y_{i t}$ wields no influence over the explanatory variable $w_{i, t+1}$ just behind its decision, it can make some sort of influences on the explanatory variables after $t+1$, while that of (2.3) is that the decision $y_{i t}$ have no influence on the explanatory variables after its decision. In addition, it is regrettable that at this stage, author is unable to construct the valid moment conditions when $w_{i t}$ is endogenous. This would be a task for the future. matrix with $m=\sum_{t=2}^{T} m_{t}$. The (transposed) blocks

$$
z_{i t}=f_{t}\left(v_{i}^{t-2}, \psi_{i}, w_{i}^{T}\right), \text { for } t=2, \cdots, T,
$$

are the $m_{t} \times 1$ vector-valued functions of $v_{i}^{t-2}, \psi_{i}$ and $w_{i}^{T}$ at time $t$, where $m_{t}$ is number of instruments for time $t$. By using the empirical counterpart of (2.13):

$$
g_{N}(\delta)=(1 / N) \sum_{i=1}^{N}\left(z_{i}\right)^{\prime} h_{i}(\delta)
$$

and the $m \times m$ inverse of optimal weighting matrix:

$$
\Omega_{N}\left(\hat{\delta}_{1}\right)=(1 / N) \sum_{i=1}^{N}\left(z_{i}\right)^{\prime} h_{i}\left(\hat{\delta}_{1}\right)\left(h_{i}\left(\hat{\delta}_{1}\right)\right)^{\prime} z_{i},
$$

where $\hat{\delta}_{1}$ is any initial consistent estimator for $\delta$, the GMM estimator is constructed as follows:

$$
\hat{\delta}_{G M M}=\arg \min _{\delta}\left(g_{N}(\delta)\right)^{\prime}\left(\Omega_{N}\left(\hat{\delta}_{1}\right)\right)^{-1} g_{N}(\delta),
$$

where $N^{1 / 2}\left(\hat{\delta}_{G M M}-\delta_{0}\right)$ converges in distribution to the normal distribution as follows:

the moment conditions (2.13), can be written by using $\delta_{0}$ as follows:

$$
\Omega\left(\delta_{0}\right)=\mathrm{E}\left[\left(z_{i}\right)^{\prime} h_{i}\left(\delta_{0}\right)\left(h_{i}\left(\delta_{0}\right)\right)^{\prime} z_{i}\right],
$$

where it should be noted that (2.16) is the empirical counterpart of (2.19) if $\hat{\delta}_{1}$ is replaced by $\delta_{0}$ and $N^{1 / 2} g_{N}\left(\delta_{0}\right) \stackrel{d}{\rightarrow} \mathrm{N}\left(0, \Omega\left(\delta_{0}\right)\right)$. Further, the first derivative of (2.13) with respect to $\delta$ for $\delta_{0}$ is as follows:

$$
D\left(\delta_{0}\right)=\left.\left(\partial \mathrm{E}\left[\left(z_{i}\right)^{\prime} h_{i}(\delta)\right] / \partial \delta\right)\right|_{\delta=\delta_{0}} .
$$

It is conceivable that the discussions for the GMM estimator based on the HTD transformation could be permitted to be conducted on the basis of numbers of observations for which $\left(\Delta y_{i t}\right)^{2}=1$ instead of $N$, on the grounds that observations except for those for which $\left(\Delta y_{i t}\right)^{2}=1$ make no direct contribution to estimating $\delta$. 
In this case, $\quad \bar{M}=(1 /(T-1)) \sum_{t=2}^{T} M_{t}$ is expediently used instead of $N$ in this section, where $M_{t}$ is number of observations for which $\left(\Delta y_{i t}\right)^{2}=1$ at time $t$.

\section{Link between CMLE and GMM Estimator}

The discussion here is conducted for the case of two periods (i.e. $t-1$ and $t$ ). It is shown in this section that the GMM estimator opting for an instrument is identical to the CMLE in this case.

First, the GMM estimator is presented. With $h_{i}(\delta)=h_{i t}(\delta)$ and $z_{i}=z_{i t}=\Delta w_{i t} / 2$ (both of which are scalars), Equation (2.13) turns to

$$
\mathrm{E}\left[\left(\Delta w_{i t} / 2\right) h_{i t}(\delta)\right]=0 .
$$

The moment condition (3.1) says that $\Delta w_{i t} / 2$ is used as the instrument for the HTD transformation $h_{i t}(\delta)$. The GMM estimator for $\delta$ is the just-identified one when using only the moment condition (3.1) for the two periods. This is denoted by $\hat{\delta}_{G M M}^{*}$ hereafter.

The first derivative of $h_{i t}(\delta)$ with respect to $\delta$ and the square of $h_{i t}(\delta)$ are respectively calculated as follows:

$$
\partial h_{i t}(\delta) / \partial \delta=-\left(\Delta y_{i t}\right)^{2}\left(\Delta w_{i t} / 2\right) \operatorname{sech}^{2}\left(\delta \Delta w_{i t} / 2\right)
$$

and

$$
\begin{aligned}
& \left(h_{i t}(\delta)\right)^{2}=\left(\Delta y_{i t}\right)^{2}-2 \tanh \left(\delta \Delta w_{i t} / 2\right)\left(\Delta y_{i t}\right) \\
& +\tanh ^{2}\left(\delta \Delta w_{i t} / 2\right)\left(\Delta y_{i t}\right)^{2} \\
& =\left(\Delta y_{i t}\right)^{2} \operatorname{sech}^{2}\left(\delta \Delta w_{i t} / 2\right)-2 \tanh \left(\delta \Delta w_{i t} / 2\right) h_{i t}(\delta),
\end{aligned}
$$

where the relationship that $\left(\Delta y_{i t}\right)^{n}=\left(\Delta y_{i t}\right)^{2}$ if $n$ is even and $\left(\Delta y_{i t}\right)^{n}=\Delta y_{i t}$ if $n$ is odd is used since $y_{i t}$ is binary. Using (2.19), (2.20), (3.2), and (3.3), $\Omega\left(\delta_{0}\right)$ and $D\left(\delta_{0}\right)$ for (3.1) are respectively calculated as follows:

$$
\begin{aligned}
& \Omega^{*}\left(\delta_{0}\right)=\mathrm{E}\left[\left(h_{i t}\left(\delta_{0}\right)\right)^{2}\left(\Delta w_{i t} / 2\right)^{2}\right] \\
& =(1 / 4) \mathrm{E}\left[\left(\Delta y_{i t}\right)^{2}\left(\Delta w_{i t}\right)^{2} \operatorname{sech}^{2}\left(\delta_{0} \Delta w_{i t} / 2\right)\right],
\end{aligned}
$$

where $\mathrm{E}\left[\tanh \left(\delta_{0} \Delta w_{i t} / 2\right) h_{i t}\left(\delta_{0}\right)\left(\Delta w_{i t}\right)^{2}\right]=0$ is used, which is obtained from (2.11), and

$$
\begin{aligned}
& D^{*}\left(\delta_{0}\right)=\mathrm{E}\left[\left(\Delta w_{i t} / 2\right)\left(\partial h_{i t}(\delta) /\left.\partial \delta\right|_{\delta=\delta_{0}}\right)\right] \\
& =-(1 / 4) \mathrm{E}\left[\left(\Delta y_{i t}\right)^{2}\left(\Delta w_{i t}\right)^{2} \operatorname{sech}^{2}\left(\delta_{0} \Delta w_{i t} / 2\right)\right] .
\end{aligned}
$$

Looking at (3.4) and (3.5), it can be seen that

$$
\Omega^{*}\left(\delta_{0}\right)=-D^{*}\left(\delta_{0}\right) \text {. }
$$

In addition, the relationship (2.18) is also applicable to the just-identified estimator (see pp. 486-487 in Hayashi,
2000, [23]). Therefore, it follows from (2.18) and (3.6) that the following relationship holds for $\hat{\delta}_{G M M}^{*}$ :

$$
N^{1 / 2}\left(\hat{\delta}_{G M M}^{*}-\delta_{0}\right) \stackrel{d}{\rightarrow} \mathrm{N}\left(0,-\left(1 / D^{*}\left(\delta_{0}\right)\right)\right) .
$$

Lee (2002, pp. 84-87) [24] elucidates the equality conceptually identical to (3.6) in the context of the CMLE to be hereafter described. In addition, Bonhomme (2012) [25] demonstrates that the conditional moment restriction which he proposes for the fixed effects logit model can give birth to the unconditional moment condition identical to (3.1).

Next, the conventional CMLE proposed by Chamberlain (1980) [1] is presented for the two periods as follows:

$$
\hat{\delta}_{C M L}^{*}=\arg \max _{\delta} L(\delta),
$$

where $L(\delta)=\sum_{i=1}^{N} l_{i t}(\delta)$. Referring to Wooldridge (2002, pp. 490-492) [26], the logarithm of probability composing the conditional log-likelihood function for the two-periods fixed effects logit model is written as follows, with $\pi_{i t}(\delta)=\exp \left(\delta \Delta w_{i t}\right) /\left(1+\exp \left(\delta \Delta w_{i t}\right)\right)$ :

$$
l_{i t}(\delta)=\vartheta_{i t}\left(\varsigma_{i t} \ln \left(\pi_{i t}(\delta)\right)+\left(1-\varsigma_{i t}\right) \ln \left(1-\pi_{i t}(\delta)\right)\right),
$$

where $\vartheta_{i t}=1$ if $y_{i, t-1}+y_{i t}=1$ and $\vartheta_{i t}=0$ otherwise, while $\varsigma_{i t}=1$ if $y_{i, t-1}=0$ and $y_{i t}=1$ and $\varsigma_{i t}=0$ if $y_{i, t-1}=1$ and $y_{i t}=0$. In (3.9), $\pi_{i t}(\delta)$ stands for the probability with which $y_{i t}$ takes one given $w_{i, t-1}, w_{i t}$, $\psi_{i}$ and $y_{i, t-1}+y_{i t}=1$, while $1-\pi_{i t}(\delta)$ stands for the probability with which $y_{i t}$ takes zero given $w_{i, t-1}, w_{i t}$, $\psi_{i}$ and $y_{i, t-1}+y_{i t}=1$.

The first-order condition of $L(\delta)$ is

$$
\partial L(\delta) / \partial \delta=\sum_{i=1}^{N} \partial l_{i t}(\delta) / \partial \delta=0
$$

with

$$
\begin{aligned}
& \partial l_{i t}(\delta) / \partial \delta \\
& =\vartheta_{i t} \Delta w_{i t}\left(\varsigma_{i t}\left(1-\pi_{i t}(\delta)\right)-\left(1-\varsigma_{i t}\right) \pi_{i t}(\delta)\right) .
\end{aligned}
$$

It is corroborated from (3.10) with (3.11) that the first-order condition of $L(\delta)$ divided by $N$ is the empirical counterpart of the moment condition (3.1) for the GMM estimator. The second-order derivative of $L(\delta)$ with respect to $\delta$ is written as

$$
\begin{aligned}
& \partial^{2} L(\delta) / \partial \delta^{2} \\
& =\sum_{i=1}^{N}-\vartheta_{i t}\left(\Delta w_{i t}\right)^{2} \pi_{i t}(\delta)\left(1-\pi_{i t}(\delta)\right) .
\end{aligned}
$$

Taking notice of the fact that $\operatorname{sech}^{2}\left(\delta \Delta w_{i t} / 2\right)=4 \pi_{i t}(\delta)\left(1-\pi_{i t}(\delta)\right)$, it is evident that if $\delta$ is replaced by $\delta_{0},(3.12)$ divided by $N$ is the empirical counterpart of (3.5) and accordingly identical to $-\Omega^{*}\left(\delta_{0}\right)$ from (3.6). Therefore, the following relationship holds for $\hat{\delta}_{C M L}^{*}$ : 


$$
N^{1 / 2}\left(\hat{\delta}_{C M L}^{*}-\delta_{0}\right) \stackrel{d}{\rightarrow} \mathrm{N}\left(0,-\left(1 / D^{*}\left(\delta_{0}\right)\right)\right) .
$$

Judging from the above, it is ascertained that for the two periods the conventional CMLE for the fixed effects logit model is identical to the GMM estimator selecting $\Delta w_{i t} / 2$ as the instrument for the HTD transformation.

To make doubly sure, the integration of $\left(\Delta w_{i t} / 2\right) h_{i t}(\delta)$ with respect to $\delta$ is conducted:

$\int\left(\Delta w_{i t} / 2\right) h_{i t}(\delta) \mathrm{d} \delta$

$=\delta\left(\Delta w_{i t} / 2\right) \Delta y_{i t}-\left(\Delta y_{i t}\right)^{2} \ln \left(\cosh \left(\delta \Delta w_{i t} / 2\right)\right)+C$,

where $C$ is the constant of integration. With $C=-\left(\Delta y_{i t}\right)^{2} \ln (2)$ for (3.14), the logarithm of probability (3.9), which composes the conditional log-likelihood function for the two-periods fixed effects logit model, is compactly rewritten as

$$
\begin{aligned}
& l_{i t}(\delta) \\
& =\delta\left(\Delta w_{i t} / 2\right) \Delta y_{i t}-\left(\Delta y_{i t}\right)^{2} \ln \left(2 \cosh \left(\delta \Delta w_{i t} / 2\right)\right)
\end{aligned}
$$

The exponential of $l_{i t}(\delta)$ in (3.15), which is equivalent to (3.9), represents the probability density when the restriction $\left(\Delta y_{i t}\right)^{2}=1$ is imposed. In this case, number of observations for which $\left(\Delta y_{i t}\right)^{2}=1$ is used instead of $N$ in this section and therefore $\hat{\delta}_{C M L}^{*}$, which is equivalent to $\hat{\delta}_{G M M}^{*}$, could be interpreted as being the asymptotically efficient estimator. This is because the CramérRao inequality is applicable in this case.

Incidentally, Abrevaya (1997) [27] shows that for the fixed effects logit model, a scale-adjusted ordinary maximum likelihood estimator is equivalent to the CMLE for the case of two periods.

\section{Monte Carlo}

In this section, some Monte Carlo experiments are conducted to investigate the small sample performance of the GMM estimator for the fixed effects logit model described in Section 2. The experiments are implemented by using an econometric software TSP version 4.5 (see Hall and Cummins, 2006, [28]).

The data generating process (DGP) is as follows:

$$
\begin{aligned}
& y_{i t}= \begin{cases}1 & \text { if } p_{i t}>u_{i t} \\
0 & \text { otherwise }\end{cases} \\
& p_{i t}=\exp \left(\psi_{i}+\delta w_{i t}\right) /\left(1+\exp \left(\psi_{i}+\delta w_{i t}\right)\right), \\
& u_{i t} \sim \mathrm{U}(0,1), \\
& w_{i t}=\alpha w_{i, t-1}+\imath \psi_{i}+\zeta_{i t}, \\
& w_{i 1}=(1 /(1-\alpha)) \imath \psi_{i}+\left(1 /\left(1-\alpha^{2}\right)^{(1 / 2)}\right) \zeta_{i 1}, \\
& \psi_{i} \sim \mathrm{N}\left(0, \sigma_{\psi}^{2}\right) ; \quad \zeta_{i t} \sim \mathrm{N}\left(0, \sigma_{\zeta}^{2}\right) .
\end{aligned}
$$

In the DGP, values are set to the parameters $\delta, \alpha$, $\iota, \sigma_{\psi}^{2}$ and $\sigma_{\zeta}^{2}$. The experiments are carried out with the cross-sectional sizes $N=100,500$ and 1000 , the numbers of time periods $T=4,8$ and 25 , and the number of replications $T R=1000$.

In the experiments, the GMM estimator based on the HTD transformation selects $\Delta w_{i t}$ as the instruments for the transformation $h_{i t}(\delta)$. That is, the $\operatorname{GMM}(\mathrm{HTD})$ estimator uses the vector of moment conditions (2.13) with $z_{i t}=\Delta w_{i t}$, which is able to be written piecewise as follows:

$$
\mathrm{E}\left[\Delta w_{i t} h_{i t}(\delta)\right]=0, \text { for } t=2, \cdots, T .^{8}
$$

As a control, another GMM estimator is used, which employs the following moment conditions disregarding the unobservable heterogeneity:

$$
\mathrm{E}\left[w_{i t} \varpi_{i t}(\delta)\right]=0 \text {, for } t=1, \cdots, T .
$$

where $\varpi_{i t}(\delta)=\exp \left(\delta w_{i t}\right) /\left(1+\exp \left(\delta w_{i t}\right)\right)$. The GMM (LgtLev) estimator (i.e. the level GMM estimator for the logit model) for $\delta$ is inconsistent due to the ignorance of the fixed effects.

The Monte Carlo results are exhibited in Table 1. The settings of values of the parameters for the explanatory variables $w_{i t}$ are the same as those used by Blundell et al. (2002) [21] for count panel data model. The small sample property of the GMM(HTD) estimator can be said to be preferable and their bias and rmse (root mean squared error) decrease as the cross-sectional size $N$ increases, which is the reflection of the consistency. In contrast, the sizable downward bias and rmse for the (inconsistent) GMM(LgtLev) estimator remain at virtually constant levels when $N$ increases. As is seen from comparisons among Simulations (a4), (a8) and (a25), among Simulations (b4), (b8) and (b25), and Simulations (c4), (c8) and (c25) for the GMM(HTD) estimator, the small sample performance of the GMM(HTD) estimator is better off as the number of time periods increases, reflecting the substantive increase of sample size. Furthermore, comparisons among Simulations (a4), (b4) and (c4), among Simulations (a8), (b8) and (c8), and among Simulations (a25), (b25) and (c25) for the GMM(HTD) estimator raise the possibility that more persistent series of the explanatory variables might bring about more deteriorated small sample performance of the GMM(HTD) estimator. ${ }^{9}$

\footnotetext{
${ }^{8}$ Since the moment conditions (4.1) are valid even under the assumptions $\mathrm{E}\left[v_{i t} \mid v_{i}^{t-1}, \psi_{i}, w_{i}^{t+1}\right]=0$ for $t=1, \cdots, T$, the usage of the GMM (HTD) estimator using the moment conditions (4.1) is generally more conservative than that of the CMLE proposed by Chamberlain (1980) [1] (see footnote 7 in section 2). The CMLE is inconsistent under the assumptions $\mathrm{E}\left[v_{i t} \mid v_{i}^{t-1}, \psi_{i}, w_{i}^{t+1}\right]=0$ for $t=1, \cdots, T$ and $T \geq 3$.

${ }^{9}$ This possibility is also pointed out in the framework of ordinary and count panel data models. For example, see Blundell and Bond (1998) [29] and Blundell et al. (2002) [21].
} 
Table 1. Monte Carlo results for the fixed effects logit model.

\begin{tabular}{|c|c|c|c|c|c|c|c|}
\hline & & $\mathrm{N}=100$ & & $\mathrm{~N}=500$ & & $\mathrm{~N}=1000$ & \\
\hline & & bias & rmse & bias & rmse & bias & rmse \\
\hline GMM(HTD) & $\delta$ & 0.08 & 0.29 & 0.02 & 0.11 & 0.01 & 0.08 \\
\hline GMM(LgtLev) & $\delta$ & -0.50 & 0.53 & -0.50 & 0.50 & -0.50 & 0.50 \\
\hline Simulation (a8): & $\mathrm{T}=8$ & & & & & & \\
\hline GMM(HTD) & $\delta$ & 0.06 & 0.19 & 0.02 & 0.08 & 0.01 & 0.05 \\
\hline GMM(LgtLev) & $\delta$ & -0.50 & 0.52 & -0.50 & 0.51 & -0.50 & 0.50 \\
\hline Simulation (a25): & $\mathrm{T}=25$ & & & & & & \\
\hline GMM(LgtLev) & $\delta$ & -0.50 & 0.51 & -0.50 & 0.50 & -0.50 & 0.50 \\
\hline Simulation (b4): & $\mathrm{T}=4$ & & & & & & \\
\hline GMM(HTD) & $\delta$ & 0.18 & 0.94 & 0.04 & 0.37 & 0.02 & 0.26 \\
\hline GMM(LgtLev) & $\delta$ & -1.01 & 1.09 & -1.00 & 1.01 & -1.00 & 1.01 \\
\hline Simulation (b8): & $\mathrm{T}=8$ & & & & & & \\
\hline GMM(HTD) & $\delta$ & 0.10 & 0.58 & 0.03 & 0.25 & 0.02 & 0.17 \\
\hline GMM(LgtLev) & $\delta$ & -1.00 & 1.08 & -1.01 & 1.02 & -1.01 & 1.01 \\
\hline Simulation (b25): & $\mathrm{T}=25$ & & & & & & \\
\hline GMM(LgtLev) & $\delta$ & -1.01 & 1.06 & -1.00 & 1.01 & -1.00 & 1.00 \\
\hline Simulation (c4): & $\mathrm{T}=4$ & & & & & & \\
\hline GMM(HTD) & $\delta$ & 0.24 & 1.63 & 0.05 & 0.65 & 0.02 & 0.46 \\
\hline GMM(LgtLev) & $\delta$ & -1.02 & 1.16 & -1.00 & 1.02 & -1.00 & 1.01 \\
\hline Simulation (c8): & $\mathrm{T}=8$ & & & & & & \\
\hline GMM(HTD) & $\delta$ & 0.09 & 1.04 & 0.02 & 0.43 & 0.02 & 0.31 \\
\hline GMM(LgtLev) & $\delta$ & -1.00 & 1.16 & -1.01 & 1.03 & -1.01 & 1.02 \\
\hline Simulation (c25): & $\mathrm{T}=25$ & & & & & & \\
\hline GMM(HTD) & $\delta$ & 0.12 & 0.59 & 0.04 & 0.24 & 0.01 & 0.16 \\
\hline GMM(LgtLev) & $\delta$ & -1.02 & 1.15 & -1.00 & 1.02 & -0.99 & 1.00 \\
\hline
\end{tabular}

Notes: 1) The parameter settings in the DGP are as follows: Simulations (a4), (a8) and (a25): $\delta=0.5 ; \alpha=0.5 ; l=0.1 ; \quad \delta_{\psi}^{2}=0.5 ; \quad \delta_{\zeta}^{2}=0.5$. Simulations (b4), (b8) and (b25): $\delta=1 ; \quad \alpha=0.9 ; \quad \imath=0 ; \quad \delta_{\psi}^{2}=0.5 ; \quad \delta_{\zeta}^{2}=0.05$. Simulations (c4), (c8) and (c25): $\left.\delta=1 ; \alpha=0.95 ; \quad l=0 ; \quad \delta_{\psi}^{2}=0.5 ; \quad \delta_{\zeta}^{2}=0.015 ; 2\right)$ No non-convergence is found in all replications; 3) In each of the GMM estimations, the initial consistent estimate is obtained by using the inverse of cross-sectional average of the products between the instruments matrix as the non-optimal weighting matrix; 4) The values of the Monte Carlo statistics are obtained using the true values of $\delta$ as the starting values in the optimization for each replication. The values of the statistics obtained using the true values are not much different from those obtained using two different types of the starting values.

\section{Average Elasticity}

For the fixed effects logit model composed of (2.1) and (2.2), the new index is constructed by using both the consistent estimator for $\delta$ described in previous sections and the average of $y_{i t}$. The average elasticity of the logit probability with respect to the exponential function of explanatory variable (which is calculated without 
relation to the fixed effects) is an appropriate index in the framework of the fixed effects logit model with time dimension being strictly fixed, where no (consistent) average marginal effect is available. ${ }^{10}$ In this section, the assumption that the variables are identically distributed among individuals is unfastened. ${ }^{11}$

With $W_{i t}=\exp \left(w_{i t}\right)$, the elasticity of the probability $p_{i t}$ with respect to the positive-valued variable $W_{i t}$ (with $\psi_{i}$ being held constant) is defined as follows:

$$
\eta_{i t}^{W}=\left(\partial p_{i t} / \partial W_{i t}\right)\left(W_{i t} / p_{i t}\right)=\delta\left(1-p_{i t}\right),
$$

for

$$
t=1, \cdots, T \text {. }
$$

Under the assumption that $N \rightarrow \infty$, the overall average elasticity of $p_{i t}$ with respect to $W_{i t}$ is calculated with the following formula:

$$
\bar{\eta}_{A}^{W}=\hat{\delta}\left(1-\bar{y}_{A}\right)
$$

where $\hat{\delta}$ is the consistent estimator for $\delta$ such that $\operatorname{plim}_{N \rightarrow \infty} \hat{\delta}=\delta$ and $\bar{y}_{A}=T^{-1} N^{-1} \sum_{t=1}^{T} \sum_{i=1}^{N} y_{i t}$. Since $p_{i t}$ is the probability and $\mathrm{E}\left[v_{i t}\right]=0$ (and accordingly variances of $v_{i t}$ are finite), it can be seen that $\operatorname{plim}_{N \rightarrow \infty} \bar{\eta}_{A}^{W}=\delta\left(1-\varphi_{A}\right)$, if

$\varphi_{A}=\lim _{N \rightarrow \infty} T^{-1} N^{-1} \sum_{t=1}^{T} \sum_{i=1}^{N} \mathrm{E}\left[p_{i t}\right]$ (which is referred to as the average logit probability in this paper). ${ }^{12}$

In addition, the cross-section average elasticity for a specific time period and the group average elasticity for a group (e.g. a gender) are able to be calculated as follows, respectively: The formula calculating the cross-section average of $p_{i t}$ with respect to $W_{i t}$ for period $t$ is

$$
\bar{\eta}_{t}^{W}=\hat{\delta}\left(1-\bar{y}_{t}\right)
$$

where $\bar{y}_{t}=N^{-1} \sum_{i=1}^{N} y_{i t}$, while that calculating the group average elasticity for group $G$ in population is

$$
\bar{\eta}_{G}^{W}=\hat{\delta}\left(1-\bar{y}_{G}\right)
$$

where $\bar{y}_{G}=T^{-1}\left(N_{G}\right)^{-1} \sum_{t=1}^{T} \sum_{j=1}^{N_{G}} y_{j t}^{G}$ with subscript $j$ denoting the member of group $G, N_{G}$ being number of individual units belonging to group $G$, and $y_{j t}^{G}$ being the binary dependent variable for the individual $j$

\footnotetext{
${ }^{10}$ Frequently, the explanatory variables in the fixed effects logit model are logarithmically transformed.

${ }^{11}$ In this case, (2.19) and (2.20) in Section 2 are replaced by $\Omega\left(\delta_{0}\right)=\lim _{N \rightarrow \infty} N^{-1} \sum_{i=1}^{N} \mathrm{E}\left[\left(z_{i}\right)^{\prime} h_{i}\left(\delta_{0}\right)\left(h_{i}\left(\delta_{0}\right)\right)^{\prime} z_{i}\right]$ and $D\left(\delta_{0}\right)=\left.\lim _{N \rightarrow \infty} N^{-1} \sum_{i=1}^{N}\left(\partial \mathrm{E}\left[\left(z_{i}\right)^{\prime} h_{i}(\delta)\right] / \partial \delta\right)\right|_{\delta=\delta_{0}}$, respectively. The same is applied to (3.4) and (3.5) in Section 3.

${ }^{12} \mathrm{Just}$ in case, it is assumed that both $\mathrm{E}\left[p_{i t}\right]$ and $\mathrm{V}\left[p_{i t}\right](<\infty)$ exist for each $i$ and $t$. However, author thinks that it seems that this assumption is satisfied in any case.
}

appertaining to group $G$ at period $t$.

\section{Conclusion}

This paper proposed the hyperbolic tangent differencing (HTD) transformation for the fixed effects logit model, with the intention of ruling out the fixed effects. The consistent GMM estimator was constructed by using the HTD transformation. The equivalence of the GMM estimator opting for an instrument and the CMLE proposed by Chamberlain (1980) [1] was revealed for the case of two periods. Then, the Monte Carlo experiments indicated the desirable small sample property of the GMM estimator based on the HTD transformation. In addition, the average elasticity of the logit probability with respect to the exponential function of explanatory variable was proposed, which is an appropriate index from the point of view that it is able to be calculated without the fixed effects. Both of the simple estimator and index will facilitate empirical researchers exploring the binary choice panel data model.

\section{REFERENCES}

[1] G, Chamberlain, "Analysis of Covariance with Qualitative Data," Review of Economic Studies, Vol. 47, No. 1, 1980, pp. 225-238. doi:10.2307/2297110

[2] J. Neyman and E. L. Scott, "Consistent Estimates Based on Partially Consistent Observations," Econometrica, Vol. 16, No. 1, 1948, pp. 1-32.

[3] G, Rasch, "Probabilistic Models for Some Intelligence and Attainment Tests," The Danish Institute for Educational Research, 1960.

[4] G, Rasch, "On General Laws and the Meaning of Measurement in Psychology," Preceeding of the 4th Berkeley Symposium on Mathematical Statistics and Probability, Vol. 4, 1961, pp. 321-333.

[5] B. E. Honoré and E. Kyriazidou, "Panel Data Discrete Choice Models with Lagged Dependent Variables," Econometrica, Vol. 68, No. 4, 2000, pp. 839-874. doi:10.1111/1468-0262.00139

[6] C. Hsiao, "Analysis of Panel Data," 2nd Edition, Cambridge University Press, Cambridge, 2003.

[7] A. Thomas, "Consistent Estimation of Binary-Choice Panel Data Models with Heterogeneous Linear Trends," Econometrics Journal, Vol. 9, No. 3, 2006, pp. 177-195. doi:10.1111/j.1368-423X.2006.00181.x

[8] J. Hahn and W. Newey, "Jackknife and Analytical Bias Reduction for Nonlinear Panel Models," Econometrica, Vol. 72, No. 4, 2004, pp. 1295-1319. doi:10.1111/j.1468-0262.2004.00533.x

[9] D. R. Cox and N. Reid, "Parameter Orthogonality and Approximate Conditional Inference," Journal of the Royal Statistical Society, Series B, Vol. 49, No. 1, 1987, pp. 1-39.

[10] T. Lancaster, "Orthogonal Parameters and Panel Data," 
Review of Economic Studies, Vol. 69, No. 3, 2002, pp. 647-666. doi:10.1111/1467-937X.t01-1-00025

[11] M. Arellano, "Discrete Choices with Panel Data," Investigaciones Económicas, Vol. 27, No. 3, 2003, pp. 423-458. doi:10.2139/ssrn.261048

[12] M. Arellano and S. Bonhomme, "Robust Priors in Nonlinear Panel Data Models," Econometrica, Vol. 77, No. 2, 2009, pp. 489-536. doi:10.3982/ECTA6895

[13] J, Carro, "Estimating Dynamic Panel Data Discrete Choice Models with Fixed Effects," Journal of Econometrics, Vol. 140, No. 2, 2007, pp. 503-528. doi:10.2139/ssrn.384021

[14] I. Fernández-Val, "Fixed Effects Estimation of Structural Parameters and Marginal Effects in Panel Probit Models," Journal of Econometrics, Vol. 150, No. 1, 2009, pp. 7185. doi:10.1016/i.jeconom.2009.02.007

[15] T. A. Severini, "An Approximation to the Modified Profile Likelihood Function," Biometrika, Vol. 85, No. 2, 1998, 403-411. doi:10.1093/biomet/85.2.403

[16] L. Pace and A. Salvan, "Adjustments of the Profile Likelihood from a New Perspective," Journal of Statistical Planning and Inference, Vol. 136, No. 10, 2006, pp. 3554-3564. doi:10.1016/j.jspi.2004.11.016

[17] A. Bester and C. Hansen, "A Penalty Function Approach to Bias Reduction in Nonlinear Panel Models with Fixed Effects," Journal of Business and Economic Statistics, Vol. 27, No. 2, 2009, pp. 131-148. doi:10.1198/jbes.2009.0012

[18] M. Arellano and J. Hahn, "Understanding Bias in Nonlinear Panel Models: Some Recent Developments," In: R. Blundell, W. Newey and T. Persson, Eds., Advances in Economics and Econometrics, Cambridge University Press, Cambridge, 2007, pp. 381-409.
[19] C. Hsiao, "Longitudinal Data Analysis," In: S. N. Durlauf and E. B. Blume, Eds., Microeconometrics, Palgrave and Macmillan, Basingstoke, 2010, pp. 89-107.

[20] L. P. Hansen, "Large Sample Properties of Generalized Method of Moments Estimators," Econometrica, Vol. 50, No. 4, 1982, pp. 1029-1054.

[21] R, Blundell, R. Griffith and F. Windmeijer, "Individual Effects and Dynamics in Count Data Models," Journal of Econometrics, Vol. 108, No. 1, 2002, pp. 113-131. doi:10.1016/S0304-4076(01)00108-7

[22] A. C. Cameron and P. K. Trivedi, "Microeconometrics: Methods and Applications," Cambridge University Press, Cambridge, 2005.

[23] F. Hayashi, "Econometrics," Princeton University Press, Princeton, 2000.

[24] M. J. Lee, "Panel Data Econometrics," Academic Press, London, 2002.

[25] S. Bonhomme, "Functional Differencing," Econometrica, 2012, in Press.

[26] J. M. Wooldridge, "Econometric Analysis of Cross-Section and Panel Data," MIT Press, Cambridge, 2002.

[27] J. Abrevaya, "The Equivalence of Two Estimators of the Fixed Effects Logit Model," Economics Letters, Vol. 55, No. 1, 1997, pp. 41-43. doi:10.1016/S0165-1765(97)00044-X

[28] B. H. Hall and C. Cummins, "TSP 5.0 User's Guide," TSP International, 2006.

[29] R. Blundell and S. Bond, "Initial Conditions and Moment Restrictions in Dynamic Panel Data Models," Journal of Econometrics, Vol. 87, No. 1, 1998, pp. 115-143. doi:10.1016/S0304-4076(98)00009-8 